\title{
BURSTING NEURONS COMMAND CONSUMMATORY FEEDING BEHAVIOR AND COORDINATED VISCERAL RECEPTIVITY IN THE PREDATORY MOLLUSK PLEUROBRANCHAEA
}

\author{
MARTHA ULBRICK GILLETTE ${ }^{2}$ AND RHANOR GILLETTE
}

\begin{abstract}
Department of Physiology and Biophysics, and The Neural and Behavioral Biology Program, University of Illinois, Urbana, Illinois 61801
\end{abstract}

Received December 13, 1982; Revised March 18, 1983; Accepted April 4, 1983

\begin{abstract}
The identification of central neuronal loci that command complex and coordinated behavioral expression offers the opportunity to study basic neural mechanisms that determine whether and under what conditions a specific behavior is expressed. It was previously shown in the isolated nervous system of Pleurobranchaea that the bilaterally paired ventral white cells (VWCs) of the buccal ganglion drive cyclic motor output in the neural network recorded in cut nerve roots to the buccal mass during prolonged endogenous burst episodes (Gillette, R., M. U. Gillette, and W. J. Davis (1978) Soc. Neurosci. Abstr. 4: 1210, (1980) J. Neurophysiol. 43: 669-685). Such burst episodes are also inducible by cAMP and its agonists (Gillette, M. U., R. Gillette, and W. J. Davis (1978) Soc. Neurosci. Abstr. 4: 1209; Gillette, R., M. U. Gillette, and W. J. Davis (1982) J. Comp. Physiol. 146: 461-470). The behavioral significance of the activity driven by the VWCs and the nature of their normal extrinsic activators has been a subject for speculation (Gillette, R., M. U. Gillette, and W. J. Davis (1978) Soc. Neurosci. Abstr. 4: 1210, (1980) J. Neurophysiol. 43: 669-685; McClellan, A. D. (1980) Ph.D. thesis, Case Western Reserve University; Croll, R. P., and W. J. Davis (1982) J. Comp. Physiol. 147: 143-153) but has remained to be established by exact specification of cell activity during behavior.

In an anterior hemi-animal preparation, which responds to appetitive and emetic stimuli with appropriate behavior, appetitive (food) stimuli, but not emetic substances, activate the previously quiescent VWCs. This activation is characterized by slow depolarization of the membrane potential accompanied by short rhythmic bursts (5 to 8 spikes); it culminates in a prolonged burst cpisode (sustained depolarization and spiking at 3 to $5 \mathrm{~Hz}$ for 0.7 to $5.0 \mathrm{~min}$ ). Activation of a VWC by squid homogenate resembles in detail the activation of the cell by cAMP and its agonists (M. Gillette, submitted for publication). Appetitive substances can activate the VWCs via stimulation of the oral veil, but they are most effective in the buccal cavity.

During the prolonged burst episode activated by squid homogenate, at the time the spike has broadened appreciably, the VWCs begin to drive the extreme retraction/protraction cycles of the radula that are characteristic of feeding behavior. This is consistent with earlier work which demonstrated that spike broadening during the burst is obligatory for driving motor output in the isolated nervous system (Gillette, R., M. U. Gillette, and W. J. Davis (1980) J. Neurophysiol. 43: 669-685). Simultaneously, in concerted action via their peripheral axons, the VWCs stimulate opening of a sphincter-like buccal-esophageal constriction, as well as marked longitudinal contraction and circular dilation of the esophagus, which is a food storage organ. During prolonged VWC burst episodes, solid food material is progressively moved from the buccal cavity into this shortened esophagus by the extreme retraction/protraction movements of the radula, confirming the ingestive nature of this motor pattern.

Emetic substances applied to the oral veil or buccal cavity were never observed to produce extensive or prolonged spike activity, nor did they result in bursting of the VWCs. Emetic stimuli produce cyclic buccal mass movements distinctly different from feeding which we interpret as
\end{abstract}

${ }^{1}$ This work was supported by a research grant from the American
Philosophical Society to M. U. G. and National Science Foundation
Grant BNS-79-18329 to R. G. We thank Dr. C. L. Prosser, J. London,
D. Green, Dr. M. P. Morse, and Dr. F. Delcomyn for helpful discussion
and critical reading of the manscript; Friday Harbor Laboratories,
University of Washington for facilities used in some studies; and especially Dr. Vera Fretter for her early guidance in molluscan biology.

${ }^{2}$ To whom correspondence should be addressed, at Department of Physiology and Biophysics, 524 Burrill Hall, University of Illinois, Urbana, IL 61801. 
egestion. Such movements are accompanied by rhythmic oscillations in the membrane potential of the VWC, but the dominating result is hyperpolarization, i.e., long-term inhibition.

The data support a role for the VWCs in the animal's response to the arrival of food in the buccal cavity such that the VWCs command the motor network output of vigorous ingestive behavior, coordinated with visceral receptivity for a large meal.

Many complex behaviors consist of a sequence of movements unique to the situation as well as stereotyped fixed action and cyclic motor patterns. Feeding is one such behavior which can generally be divided into two distinct phases. The appetitive phase, during which the organism detects the food stimulus orients, positions itself for feeding, and contacts the food, involves movements which change with situational variables as well as with the behavioral history of the animal. In contrast, the consummatory phase, during which the food is actually ingested, displays movements which are relatively stereotyped.

Analysis of feeding behavior in mollusks of dissimilar feeding habits has revealed general features common to Pleurobranchaea (Davis and Mpitsos, 1971), Aplysia (Kupfermann, 1974a, b), Limax (Gelperin et al., 1978), and Tritonia (Willows, 1978). Each species displays appetitive movements that are variable in detail but generally appropriate to tracking the particular type of food it eats. The consummatory phase in each is distinctive, but a common feature is stereotyped, cyclic movements of the buccal mass.

In the carnivorous and predatory marine mollusk, Pleurobranchaea californica, food capture involves a biting proboscis extension, the bite/strike, during which the food is grasped in the mouth. This is followed by cyclic protraction/retraction movements of the radula/odontophore of the buccal mass, during which food is ingested (Davis and Mpitsos, 1971). Ingestion is accomplished by protraction of the radular sac, which pushes the radula forward to grasp the food, and retraction, which pulls the food into the buccal cavity. The result is that food accumulates in the esophagus (Hirsch, 1915). This cyclic motor pattern is produced by alternating bursts of activity in protraction and retraction motor neurons in the buccal ganglion, which are driven by a feeding oscillator in the buccal ganglion (Davis et al., 1973).

Recent work has turned to examining the critical network loci at which this cyclic motor pattern is controlled. Gillette et al. (Gillette, R., et al., 1978b, 1982c) described the paracerebral neurons of the cerebral ganglion which may command feeding motor output through their position in a powerful positive feedback loop with the feeding oscillator; they are synaptically driven by the oscillator to re-excite it. A second potential command locus was identified in the ventral white cells (VWCs) of the buccal ganglion, which command cyclic motor output to the buccal mass by an intrinsic mechanism, the self-sustained capacity to burst (Gillette, R., et al., 1978a, 1980); the behavioral context of the activation of these neurons is the subject of this paper.

The VWCs are a single pair of electrically coupled neurons in the buccal ganglion whose activity can initiate or intensify cyclic motor neuron output monitored in the cut nerve roots of the isolated buccal ganglion of Pleurobranchaea (Gillette et al., 1980). The VWCs' command function derives from an inducible property, the capacity to fire in high intensity and prolonged burst episodes. The ability of the VWCs to drive motor output is specifically dependent upon a pronounced increase in the duration of the action potential (spike broadening) which proceeds from high frequency ( 4 to $5 \mathrm{~Hz}$ ) repetitive firing early in the burst episode. In isolated ganglia, quiescent VWCs can be induced to fire prolonged bursts and display attendant spike broadening by agents that elevate intracellular cAMP, which may act as an intrinsic modulator of these neurons (Gillette, M. U., et al., 1978; Gillette, R., et al., 1982b).

Cyclic motor activity in isolated buccal ganglia has been associated with both ingestive and cgestive behaviors (Davis et al., 1973; McClellan, 1979, 1980; Croll and Davis, 1982). Both are stereotyped behaviors involving cyclic movements of the buccal mass. In light of the potent effect VWC bursting has on the motor output of the buccal ganglion, we undertook this study on an anterior hemi-animal preparation as a first step in identifying pathways presynaptic to the VWCs. We sought to establish the nature of chemosensory stimuli which by way of the sensory network are extrinsic activators of the VWCs. We then examined how these activators relate to the behavioral output of these neurons to the buccal mass.

In this paper we demonstrate that appetitive substances applied to the oral veil or buccal cavity activate the VWCs to produce the prolonged burst episodes which drive the buccal motor network; emetic substances at these sites cause long-term inhibition in the VWCs. We demonstrate further that the prolonged burst episode activated by food drives extreme retraction/protraction cycles in the radula and that activation of this motor pattern by VWC bursting moves food progressively from the buccal cavity to the esophagus. High levels of activity in the VWCs also alter the morphology of the esophagus so as to facilitate efficient and rapid filling. Thus, the VWCs are neurons which have a major role in driving vigorous ingestion, and whose command ability is specifically and appropriately induced by appetitive stimuli.

\section{Materials and Methods}

Pleurobranchaea californica werc supplied by Rimmon C. Fay, Pacific Bio-Marine, Venice, CA, and were held without feeding for 1 to 2 weeks at $14^{\circ} \mathrm{C}$ in our sea water system. Animals were pretested for their ability to respond to appetitive stimuli (squid homogenized in $1 \mathrm{vol}$ of sea water $(\mathrm{w} / \mathrm{v})$, designated $\mathrm{SH}$, or $10 \%$ Dr. Bronner's amino acid hydrolysate in sea water) and emetic stimuli (10\% EtOH or $1 \%$ Haemosol in sea water). Response to these stimuli did not vary with simply making them up 
in sea water or adjusting their final $\mathrm{pH}$ to 7.5 . As the $\mathrm{pH}$ did not affect the response, it was not adjusted in most experiments. All animals used in the study responded to appetitive stimuli with a vigorous bite/ingestion sequence. Emetic stimuli were assessed by their ability to interrupt ingestion of a string coupled with an appetitive stimulus (to assess direction of net flow) and induce immediate egestion of the string.

Immediately after behavioral testing, an anterior hemi-animal preparation (consisting of head, buccal mass, esophagus, central nervous system, anterior foot, mantle, and gill) was made in order to reduce pedal locomotion which would dislodge the intracellular electrode and facilitate access to the VWC while minimally disiurbing the relevant structures (Fig. 1). The extensive acid gland was removed and the acid duct was ligated to prevent $\mathrm{H}_{2} \mathrm{SO}_{4}$ secretion (Hirsch, 1915) from acidifying the sea water in the experimental chamber. The hemianimal was then rinsed three times in 0.5- to 1.0-liter volumes of sea water. This preparation was pinned out on Sylgard (Fig. 1) in a large Lucite chamber in $14^{\circ} \mathrm{C}$ filtered artificial sea water from our system, adjusted to $\mathrm{pH}$ 7.5. To prevent acidification of the chamber sea water by the animal's surface mucus, which we found to be $\mathrm{pH}$ 2 , a one-sixth volume was changed at least every half hour. These conditions were found necessary to maintain the preparation's health and responsiveness throughout the experiment.

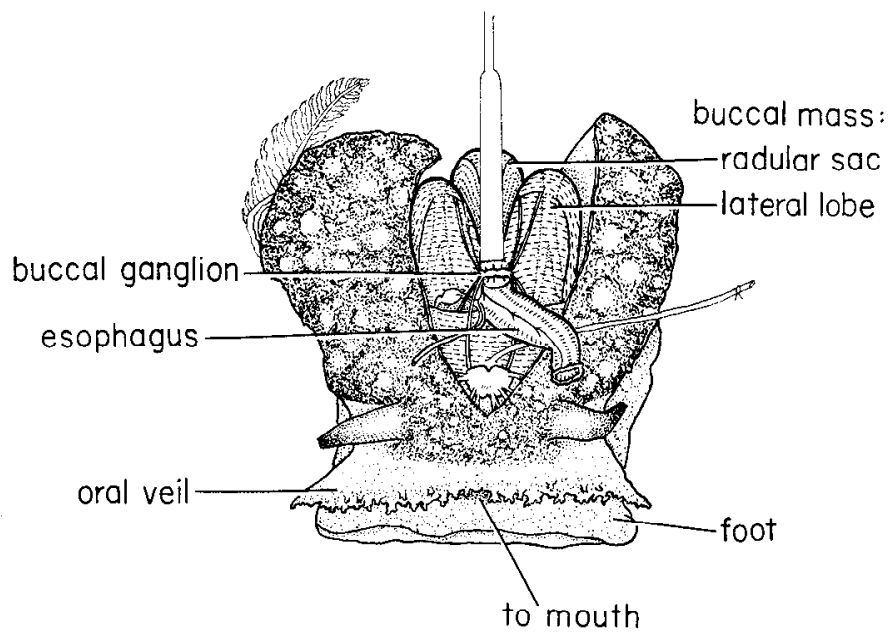

Figure 1. The hemi-animal preparation. A dorsal incision is made in the animal to the mid-rhinophore region, and posterior structures are removed. The cerebral ganglion with all of its roots intact lies just posterior to the anterior end of the incision. The esophagus is cut just anterior to the gastroesophageal sphincter and reflected with the cut end forward so that the buccal ganglion which lies beneath it can be seen. The buccal ganglion is inverted to present the ventral surface and is pinned through the connective tissue to a micromanipulated platform. Nerve roots emanating from the buccal ganglion, starting with the most anteriomedial in this illustration, include the stomatogastric nerve, innervating the esophagus and salivary duct, the cerebrobuccal connective to the cerebral ganglion and roots 3,2 , and 1 to the buccul mass. The radula and odontophore of the buccal mass lie within the radular sac directly beneath the platform. The mouth is obscured by the oral veil. Visible as a ligated tube on the right is the main acid duct, and on the left is the feathery gill. $B a r=1 \mathrm{~cm}$.
The head of the animal bearing the chemosensory structures was isolated by a Parafilm collar which fit snugly around the body behind the rhinophores and extended across the chamber. The collar acted as a physical barrier between the anterior chamber, where test substances were introduced, and the posterior chamber, where nervous tissue was exposed. Test substances were introduced by pipette over the oral veil or into the buccal cavity, or by cannula inserted directly into the buccal cavity. The buccal ganglion was reflected on a small micromanipulated platform to expose the ventral surface and was immobilized by pinning through the connective tissue (Fig. 1). No buccal roots or other CNS structures or roots were cut. The muscular outer sheath of connective tissue was manually dissected; the inner transparent sheath directly over each VWC was focally softened by $6 \mathrm{~min}$ exposure to $0.2 \%$ pronase in a $1-\mathrm{mm}$ diameter glass capillary lowered over the VWC soma, followed by extensive rinsing of the region. The soma of the VWC, identified by its position and whitish color, was impaled through the inner sheath by a glass microelectrode filled with $3 \mathrm{M} \mathrm{KCl}$; identification was confirmed by observing the distinctive spike waveform and its extracellularly recorded axonal spike en passant in the contralateral stomatogastric nerve (SGN). Records of electrical activity were made on a Gould 220 or 2400 chart recorder.

The buccal mass and esophagus were left unrestrained, respectively, directly beneath and above the platform on which the buccal ganglion was immobilized. They moved freely throughout the experiment and their responses to stimuli were observed directly. In some experiments anterior/posterior movements of the buccal mass were recorded by connecting the radular sac to a force-displacement transducer (Grass Instruments) coupled to a Gould 2400 chart recorder. The experiments relating esophageal shortening directly to VWC activity utilized the force transducer hooked bilaterally through the distal end of the esophagus near the cut gastroesophageal sphincter of a reduced preparation composed of buccal ganglion, buccal mass, and esophagus; the reduced preparation was used for these experiments only.

The net direction of flow of material induced by buccal mass movements during VWC bursting was assessed by inserting into the buccal cavity of the hemi-animal a squid ribbon (SR; 3 to $4 \mathrm{~mm}$ in diameter) marked at 10$\mathrm{cm}$ intervals with acrylic fingernail polish. Each SR was obtained by cutting the squid mantle into a long, continuous spiral. We found it necessary to introduce the SR just before recording from the VWC for otherwise, the animal fed for a period, then chewed off the ingested length. Movement of the SR was monitored visually and recorded cinematically during induced and spontaneous bursting. The relation of the progressive movements of the SR to the cycle of the radula was determined by stopframe analysis.

\section{Results}

\section{Response of the anterior hemi-animal preparation to chemosensory stimulation}

When the anterior hemi-animal preparation was presented with chemosensory stimuli, the head region re- 
sponded in a way appropriate to the stimulus. Appetitive stimuli ( $\mathrm{SH}$ or amino acid hydrolysate) applied to the head region produced orienting movements. Typically these consisted of extension of the oral veil toward the stimulus with sensory papillae flared, often with the tips of the oral veil curling around the food. The dense, milky suspension of squid fell from the oral veil to the floor of the preparation dish, coming in contact with the mouth region, which is also chemosensory. Frequently this stimulated vigorous buccal mass movements, although the hemi-animal was not observed to evert its proboscis, possibly due in part to the loss of hydrostatic pressure during dissection (Fretter and Graham, 1962) and to the restraint put on it in order to record from the buccal ganglion, which lies over the buccal mass. SH placed directly in the buccal cavity produced extreme anteriorposterior movements of the radula sac with attendant pumping of the suspension into the esophagus, where it passed out of the cut end.

Emetic stimuli (EtOH, Haemosol) applied to the anterior end of the preparation caused withdrawal both of sensory papillae and of the oral veil for the duration of stimulus application. Like food stimuli, emetic stimuli caused cyclic buccal mass movements when placed directly in the buccal cavity, but with major differences. The emetic stimuli failed to cause the extreme retraction phase of radular movements seen with food; instead, the lateral folds of the buccal mass moved medially in a rhythmical fashion while the radular sac moved from rest to protraction. Movement of the clear EtOH solution could not be observed. The whitish Haemosol solution was not passed through the esophagus but was egested and deposited outside the mouth after this buccal mass activity.

Neutral stimuli did not elicit either of the above responses. Touching the oral veil briefly with a glass rod caused a rapid local withdrawal of the oral veil; the oral veil was relaxed again within a few seconds of the touch. No movement of the buccal mass occurred.

Each of these responses is similar to that elicited by the various stimuli in the intact animal. These results demonstrate that the anterior hemi-animal preparation preserves the major aspects of ingestion and egestion behavior and is therefore a valid model for examining neurophysiological effects of chemosensory stimuli.

\section{Extrinsic activators of the $V W C$}

Response of the VWC to appetitive stimuli. When an appetitive substance ( $\mathrm{SH}$ or amino acid hydrolysate) was presented to a behaviorally responsive hemi-animal, the VWC invariably changed from a state of quiescence to one of activity ( 18 separate trials on 9 animals). In these hemi-animal preparations with the whole of the central neural connections undisturbed by dissection, the VWC was typically silent upon penetration by microelectrode, with only the occasional single action potential interrupting the base line (Fig. 2). This contrasts with the high level of spontaneous activity usually observed in the isolated ganglion (Gillette et al., 1980).

After establishing the unstimulated character of VWC activity for at least 20 to $30 \mathrm{~min}$, food was applied to the animal's oral veil or buccal cavity. This application of an

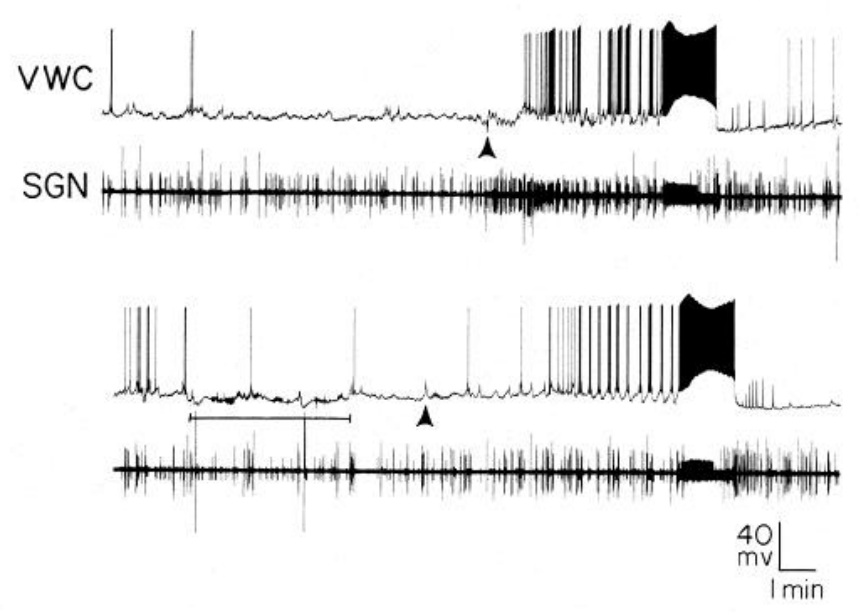

Figure 2. Excitation of the VWC by an appetitive stimulus placed in the buccal cavity (upper records) and onto the oral veil (lower records). Direct introduction via cannula of $\mathrm{SH}$ into the buccal cavity caused synaptic depolarization and phasic bursts of spikes in the VWC; the excitation culminated in a single prolonged burst episode and movement of $\mathrm{SH}$ out the cut end of the esophagus. A sustained increased in spontaneous firing rate was subsequently reduced by cannular rinsing of the buccal cavity 27 min later (bar in lower record). Thereafter, $\mathrm{SH}$ application to the oral veil initiated another burst episode, with somewhat longer latency. The spike activity of the VWC and other neurons was recorded extracellularly in the contralateral SGN.

appetitive substance activated the quiescent VWC in every animal tested $(N=9)$. Activation was characterized by depolarization of the base line, an onset of spike activity, and an increase in spike frequency. The increase in spike frequency progressed from an increase in the rate of single action potential discharges to short bursts of 3 to 8 spikes, and activation culminated in prolonged burst episodes, 0.7 to $5 \mathrm{~min}$ in duration at spike frequencies of 3 to $5 \mathrm{~Hz}$, typical of the VWC (Gillette et al., 1980). Figure 2 shows the response of the VWC to $\mathrm{SH}$ applied to the buccal cavity of the preparation immediately after the unstimulated base line was established. These intracellular records demonstrate the rapid depolarization of the base line, the appearance of short bursts, and the typical intense spike activity during the prolonged burst. After extensive rinsing of the buccal cavity, $\mathrm{SH}$ was applied to the oral veil (Fig. 2, line 2), where it also activated VWC bursting.

Although the elements of activation were qualitatively the same, the rate of progression of VWC activation to the prolonged burst episode was variable depending upon such factors as the site at which the stimulus was applied and the behavioral history of the animal (see below). Thus, records of individual stimulation trials of the VWC by appetitive substances differed in the details of activation, but the common result was that food substances at chemosensory loci activate the VWC and this stimulation culminates in the prolonged burst episodes of the VWC.

Response of the VWC to emetic stimuli. VWC responses to emetic stimuli applied to chemosensory areas fell into two classes (Fig. 3). One class was characterized by a slow, steady hyperpolarization of the membrane poten- 
A

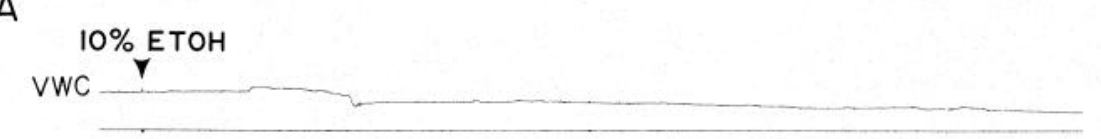

B

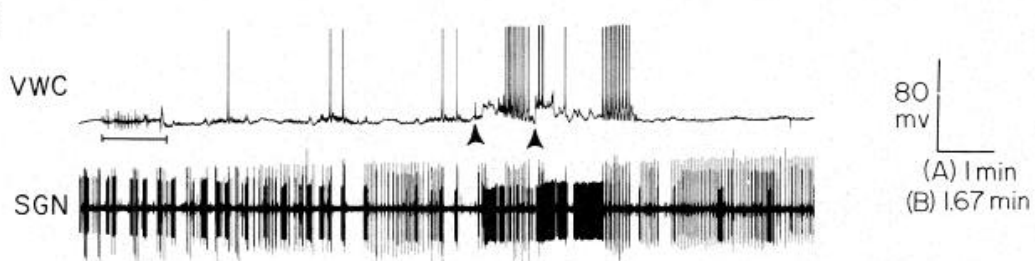

Figure 3. Responses of the VWC to emetic stimuli. Two classes of responses were observed in intracellular records of the VWC during application of emetic stimuli to chemosensory structures. A, Application of $10 \% \mathrm{EtOH}$ to the animal's oral veil (arrowhead) caused $-20-\mathrm{mV}$ hyperpolarization of the VWC in $16 \mathrm{~min}$. B, Each of two applications of $10 \% \mathrm{EtOH}$ applied to this animal's buccal cavity (arrowheads) after SH washout (bar) produced rhythmic oscillations in the membrane potential accompanied by a brief volley of spikes at the end of each stimulation. This was followed by silence lasting until stimulus washout. The extracellular record of the contralateral SGN shows intense activity in an unidentified unit, which is not the VWC.

tial with no spiking and few, if any, postsynaptic potentials (Fig. $3 A$ ). The cell remained hyperpolarized and silent, recovering gradually after stimulus washout. The second class of responses of the VWC to emetic stimuli was a rhythmic oscillation of the membrane potential for 2 to $4 \mathrm{~min}$. These oscillations occasionally included a brief flurry of spikes, but then, rather than increasing in activity, the VWC became quiet (Fig. $3 B$ ). This record shows that tonic activity in a unit in the contralateral SGN increased within $15 \mathrm{sec}$ of introduction of the stimulus during the membrane potential oscillations of the VWC, but it is clearly not the axon of the VWC, because it fires in antiphase to VWC spike activity. This finding questions studies in which tonic activity in the SGN during egestion was interpreted to be VWC activity without recording from the cell (McClellan, 1980; Croll and Davis, 1982). The functional significance of activity in this tonic unit during egestion is unknown.

The responses of the VWCs of six animals to emetic stimuli applied to the buccal cavity are summarized in Table I. Although emetic stimuli may initially produce rhythmic oscillations in the membrane potential, the general result was for them to hyperpolarize or inhibit the VWC. This effect was reversible upon stimulus washout and reapplication of food. Emetic substances were never observed to produce extensive or prolonged spike activity, nor did they result in prolonged bursting as did appetitive stimuli. These results show that the sign of activity in the $\mathrm{VWC}$ in response to emetic stimuli applied to chemosensory areas of the head is negative.

\section{Efficacy of oral veil versus buccal stimulation of the $V W C$}

Ingestion of food in other mollusks, Limax and Aplysia, has been demonstrated to involve both peripheral olfactory receptors as well as gustatory receptors of the mouth or lips (Kupfermann, 1974a, b; Gelperin et al., 1978; Reingold and Gelperin, 1980). Focal application of food at restricted sites on Pleurobranchaea's chemosensory
TABLE I

Response of the ventral white cell to emetic stimuli

Response in the Ventral White Cell Rhythmic oscillations Cell quiet, in membrane potential, Prolonged hyperpolarizes occasional spikes, (Egestion?)

\begin{tabular}{llll}
\hline $\begin{array}{l}10 \% \mathrm{EtOH} / \mathrm{sw}^{a} \\
(n=4)\end{array}$ & 2 & 2 & 0 \\
$\begin{array}{l}1 \% \text { Haemosol/sw } \\
(n=3)\end{array}$ & 1 & 2 & 0 \\
\hline${ }^{a}$ sw, sea water & & & \\
\hline
\end{tabular}

anterior structures has been shown to elicit appetitive feeding responses such as mouth opening and proboscis extension (Lee and Liegeois, 1974; Lee et al., 1974). Nonfocal application of food to large areas of the oral veil and rhinophores elicits both the appetitive and consummatory phases (Davis and Mpitsos, 1971); exposure of the mouth region to the food has not previously been controlled. We therefore determined the efficacy of appetitive stimuli at oral veil versus buccal receptors in activating the VWC.

Cannulation of the buccal cavity provided a way to selectively stimulate buccal receptors while the cannula itself prevented the access of $\mathrm{SH}$ applied at the oral veil. A length of polyethylene tubing ( $3 \mathrm{~mm}$ outside diameter) was gently worked into the mouth, sometimes with the aid of oral veil stimulation with $\mathrm{SH}$ to induce mouthopening, followed by rinsing. Under these conditions, application of $\mathrm{SH}$ to the oral veil of a cannulated animal resulted in prolonged VWC bursting in only one animal (Table II). However, in uncannulated animals, whose mouth was not occluded but simply withdrawn due to the restraint on the preparation, three of four animals demonstrated gradual increase in activity in the VWC culminating in a prolonged burst episode. In all cases, both with the mouth open and cannulated, the VWC 
TABLE II

Response of the ventral white cell to different modes of squid homogenate $(\mathrm{SH})$ stimulation

\begin{tabular}{lccc}
\hline & \multicolumn{3}{c}{ Response in the Ventral White Cell } \\
\cline { 2 - 4 } & $\begin{array}{c}\text { Increased Spike } \\
\text { Activity } \\
\text { No Prolonged } \\
\text { Bursts }\end{array}$ & $\begin{array}{c}\text { Increased Spike } \\
\text { Activity } \\
\text { A Single } \\
\text { Prolonged Burst }\end{array}$ & $\begin{array}{c}\text { Increased Spike } \\
\text { Activity } \\
\text { Recurrent }\end{array}$ \\
\hline $\begin{array}{c}\text { SH on oral veil; } \\
\text { mouth unob- } \\
\text { structed }\end{array}$ & 1 & 1 & 2 \\
$\begin{array}{c}\text { SH on oral veil; } \\
\text { mouth occluded }\end{array}$ & 5 & 1 & 0 \\
$\begin{array}{c}\text { SH in buccal cavity } \\
\text { briefly via curved } \\
\text { pipette }\end{array}$ & 0 & 3 & 0 \\
SH in buccal cavity \\
continuously via \\
cannula
\end{tabular}

demonstrated a gradual increase in activity in response to oral veil stimulation with $\mathrm{SH}$. These results suggest that although food on the oral veil excites the VWC, access of food to the buccal cavity may be important for activation of the neuron to bursting.

The efficacy of appetitive stimuli at buccal receptors was examined by introducing SH directly through the mouth into the buccal cavity either by cannula or curved pipette. When SH was introduced directly into the buccal cavity, without concurrent oral veil stimulation, $\mathrm{SH}$ invariably produced prolonged burst episodes in the VWC (14 trials in 8 animals, Table II) with a shorter latency as compared with oral veil stimulation alone. These results emphasize a more important role for buccal receptors in activating the VWC.

There is the possibility that introduction of the cannula may partially inhibit the feeding response and thus account for the low rate of VWC activation by oral veil stimulation in its presence. Nevertheless, the same amount of stimulus was applied to both the oral veil and the buccal cavity in the presence of the cannula, yet prolonged burst episodes were only stimulated by buccal application. Thus, even if the cannula caused some suppression of feeding, buccal receptors still are more effective than those on the oral veil at overcoming that suppression and activating feeding.

\section{Recurrent burst episodes in the VWC stimulated by chemosensory inputs}

Earlier observations on the VWC in isolated buccal ganglia (Gillette, R., et al., 1978a, 1980) and on VWCs tonically stimulated with isobutylmethylxanthine or cAMP analogues (Gillette, M. U., et al., 1978; Gillette, R., et al., 1982b) established that the VWC may generate recurrent burst episodes at intervals of 5 to $30 \mathrm{~min}$. However, spontaneous bursts were rarely observed in the hemi-animal ( 1 of 9 preparations). We were therefore interested to observe whether recurrent bursting occurred in VWCs stimulated by chemosensory inputs, and under what conditions this might take place. We examined VWCs stimulated with SH via four modes of stim- ulation: $\mathrm{SH}$ on the oral veil when the mouth was unobstructed; $\mathrm{SH}$ on the oral veil with the buccal cavity occluded by cannula; $\mathrm{SH}$ introduced into the buccal cavity by one squirt of a curved pipette which was then removed; and $\mathrm{SH}$ introduced by a cannula which remained in the mouth and in which SH remained after the initial introduction.

The results of this study appear in Table II. Recurrent prolonged burst episodes in the VWC occurred only in two situations: (1) when SH was applied to the oral veil with the mouth unobstructed and (2) when SH was delivered directly to the buccal cavity via cannula with some $\mathrm{SH}$ remaining in the cannula during the interburst period. In both situations the receptors of the buccal cavity are likely to be tonically stimulated. SH applied to the oral veil could be pulled into the buccal cavity over a period of time by bites and thus provide long-term exposure of the buccal receptors to food. Cannular application allows continuous exposure of buccal receptors to $\mathrm{SH}$ due to continuous leakage from the cannula; it produced recurrent bursting in each of 11 trials on 7 different animals. In contrast, brief stimulation by a single application of $\mathrm{SH}$ from the pipette produced a single burst for each trial. These results suggest that continuous exposure of buccal receptors to the appetitive stimulus for a period of time after the initial burst is necessary to initiate burst recurrence in the anterior hemi-animal.

\section{Buccal mass movements and VWC activity during chemosensory stimulation: Appetitive responses}

It was previously shown in the isolated buccal ganglion that when the VWC is phasically co-active with retraction motor neurons, as it often is preceding a prolonged burst (Fig. 2) or may be during egestion (Fig. 3), it has no effect on the output of the network. Fiffects of the VWC on the network are only detectable when the duration of the spike (spike broadening) increases by more than 3-fold as a result of such sustained repetitive firing as occurs in the prolonged burst episode (Gillette et al., 1980). We wished to establish the relationship between the VWC bursting activated by food and network output in the intact hemi-animal. We therefore looked at the correlations between chemosensory stimulation, activity in the VWC, and movements of the buccal mass.

Introduction of food into the preparation's buccal cavity initially produces characteristic rhythmic short bursts of 8 to 10 spikes riding on a slow depolarization of the VWC; during this period the radula cycles from resting position to protraction and back to rest in rhythm with each short burst, hyperpolarization, and short burst in the VWC (Fig. 4). This action might correspond to bites which capture the prey and pull it into the mouth, events which initiate the consummatory phase of feeding.

However, during the prolonged burst episodes of the VWC, the movements of the buccal mass undergo three distinguishing changes. (1) The excursion of the radula changes so that during the cycle it swings from protraction through rest position to extreme retraction (Fig. 4). The radula pauses at the retraction end of the cycle and 

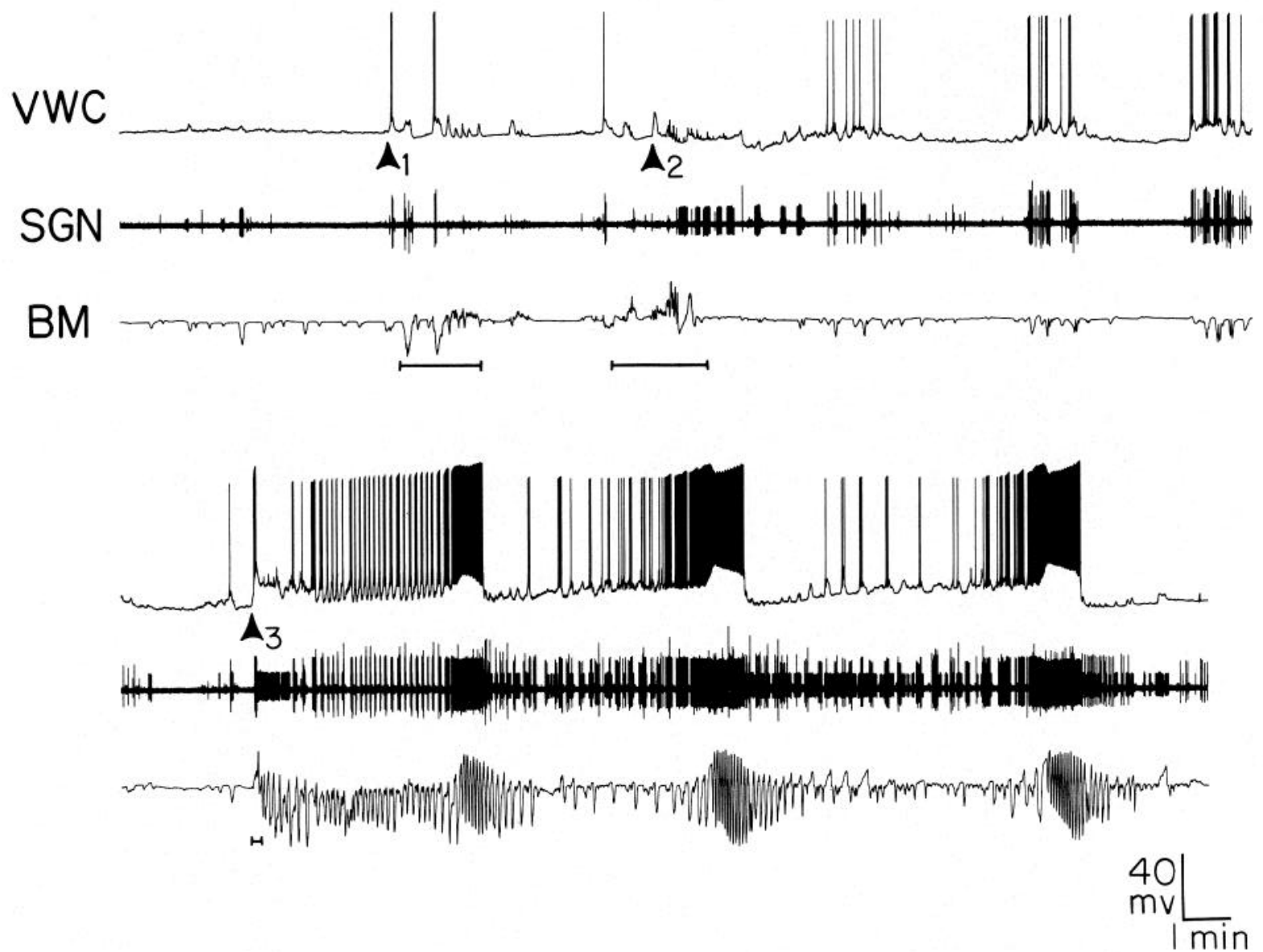

Figure 4. Buccal mass movements during VWC activity: appetitive responses. Shown are responses of the VWC (intracellular record), contralateral SGN (extracellular record), and buccal mass $(B M)$ (force transducer record). Records are continuous. SH was applied to the oral veil to induce the animal to open its mouth for cannula insertion (arrowhead 1). Placement of the cannula was tested by introducing sea water (arrowhead 2). Subsequently SH was introduced directly into the buccal cavity via the cannula (arrowhead 3). Bars mark manual disturbance of the buccal mass during manipulation of the cannula. Buccal mass movements were recorded by a force transducer hooked through the posterior cartilage of the odontophore and thus reflect only movements of this central, radula-bearing portion of the buccal mass. Downward deflections of the trace mark protraction. Further details are in the text.

is retracted fully $0.5 \mathrm{~cm}$ past rest position in a $300-\mathrm{gm}$ animal, clearly an extreme retraction position. This pause accounts for the dominance of retraction in the cycles of retraction/protraction movements driven during the prolonged VWC burst. Typically, in the distinctive rhythm cycles driven early in the burst, the duration of the retraction phase is 2 to 3 times longer than the protraction phase; the duration of retraction wanes slightly later in the VWC burst over the repeated cycles but continue to dominate. The mean ratio of the duration of protraction to total cycle time in 34 cycles driven by four VWC burst episodes was found to be $0.41 \pm 0.04$ (range 0.2 to 0.54 ) based upon measurements of the duration of movements on either side of rest in force transducer records (including those in Fig. 4). (2) The cycling rate becomes vigorous and rapid (16 to 18 cycles/ min) (Fig. 4). This is more than a 2 -fold increase over the cycling rate before the prolonged burst episode in the VWC. (3) The lateral lobes of the buccal mass, muscular elements each of which contains the chitinous extension of one of the bilateral jaws, roll forward and under the edges of the radular sac during the protraction phase of each cycle. Each of these changes was observed only during appetitive stimulation of the animal.

\section{Movement of squid ribbon during the VWC prolonged burst}

We further substantiated the relationship between VWC bursting and ingestion by monitoring the movement of a ribbon of squid mantle (SR) during stimulated and spontaneous burst episodes of the VWC. Introduction of the SR to the buccal cavity of the hemi-animal caused moderate VWC excitation in all four preparations examined in this way, but stimulated a single prolonged burst episode, accompanied by ingestion, in only two, probably because dissected animals show elevated feeding thresholds. Ingestion of the SR was induced six times in two preparations when VWC burst episodes were stimulated by providing extra stimulation with $\mathrm{SH}$, which is a more potent stimulus for feeding than whole squid flesh in both intact animals and semi-intact preparations (unpublished observations). SR ingestion was 
induced in two preparations each of five times that VWC firing was driven by injected current from the microelectrode. There was no sign of egestion in any of these trials. Results were similar in all cases, whether VWC activity was caused by food stimulation by SR, by SH, or by imposed VWC depolarization: there was net movement of the SR into the esophagus during the VWC burst so that SR accumulated there.

Stop-frame analysis of movie film shows the pattern of movement of the SR exiting the cut end of the esophagus during one cycle of such a feeding sequence (Fig. 5). These records indicate a small discontinuity in the movement of the SR during the cycle, in that there was a small retraction of the SR which most recently entered the esophagus back toward the buccal cavity during retraction of the radula. This suggests that the radular denticles still engage the newly ingested portion of squid during this phase. It seems likely that the pause at extreme retraction is when the denticles must disengage from the SR, for at the next protraction of the radula

A

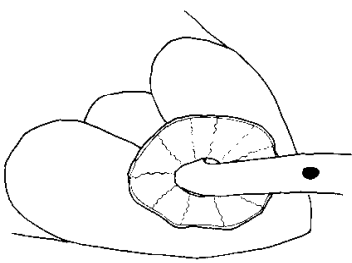

B
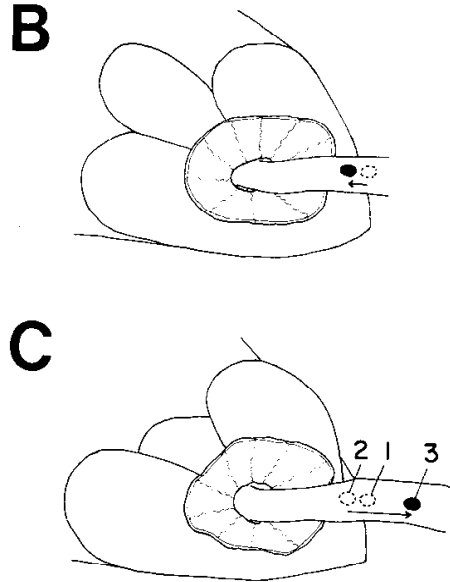

Figure 5. Ingestion of SR driven by the VWC. Tonic, repetitive firing at 4 to $5 \mathrm{~Hz}$ was driven by intracellular current injection. Line drawings were traced from stopped movie frames during one cycle of buccal mass movements. The buccal mass is viewed obliquely from above the hemi-animal pinned out as in Figure 1: anterior is to the lower right, posterior is to the upper left. The SR protrudes from the inner surface of the cut, flaired esophagus (radiating structure). The esophagus has shortened and dilated, the form it assumes during VWC bursts episodes. The movement of the SR was monitored by the relative position of a dark spot of fingernail polish. $A$, Position during extreme radular protraction. $B$, At extreme retraction of the radula, the SR has been pulled slightly back into the buccal cavity. $C$, Upon completion of a full movement cycle, the net SR movement is out of the buccal mass distally, into the esophagus. Numbers designate the order of positions of the spot in $A, B$, and $C$, respectively. $B a r=1 \mathrm{~cm}$. the newly ingested portion of squid is pushed in a looped pattern, up and forward, through the open buccal-esophageal sphincter on the anteriodorsal surface of the buccal cavity and into the esophagus where it accumulates.

\section{Relationship between VWC waveform during prolonged burst episodes and extreme buccal mass movements}

Closer inspection of the time course of the prolonged burst episode reveals a distinct relationship between the progression of the burst and the onset of extreme buccal mass movements. The buccal mass movements do not immediately change with the onset of high frequency firing $(4$ to $5 \mathrm{~Hz}$ ) of the VWC at the initiation of the burst. Rather, the time that the buccal mass first exhibits extreme retractions and increased cycling frequency during its activation by food invariably coincides with development of the burst in the VWC to a point where the undershoot of the spike is greatly attenuated. This correlation can be seen in each of the recurrent burst episodes in Figure 4 by looking for the highest point under the prolonged burst, which is actually the greatest attenuation of the undershoot, and visually drawing a vertical line down to the buccal mass record: this marks the initiation of the extreme, rapid buccal mass movements. Expanding the record at this point in the burst (Fig. 6) demonstrates that the duration of the spike has increased appreciably over the unbroadened spikes that initiate the burst (from 6 to about 75 msec measured at half-amplitude of the initial spike) (cf. Gillette et al., 1982a).

At the time that the broadened spike is driving vigorous, extreme cycling in the buccal mass through its outputs within the buccal ganglion, VWC output through its peripheral axon to the esophagus is measurably increased. Each broadened somatic spike drives two axonal spikes in the contralateral SGN (Fig. 6). Thus broadening of the spike during the high frequency repetitive firing of the prolonged burst episode functionally increases the rate of firing in the distal processes of this cell, presumably with attendant enhancement of peripheral output.

\section{Response of the esophagus during activation of the VWC by food}

We previously showed that each VWC innervates the contralateral side of the esophagus through ramifications of the contralateral SGN (Gillette et al., 1980). Soon after exiting the ganglion, the $\mathrm{SGN}$ gives off branches to the salivary duct and buccal-esophageal sphincter region. The SGN then travels along the lateral surface of the esophagus and periodically ramifies, innervating the esophagus at multiple loci (Fig. 1). In the present study two marked changes in the esophagus were observed each time that the VWC was activated to bursting by food (10 trials, 8 animals) or stimulated by depolarizing current injection (25 trials, 14 animals): (1) the buccalesophageal sphincter was tonically opened to widen the passageway from the buccal cavity to the esophagus and (2) the esophagus changed from a long narrow tube to a short wide structure as the structure dilated and the longitudinal muscles shortened (Figs. 5 and 7). These changes take place at the onset of the repetitive firing.

The relationship between VWC activity and the esoph- 

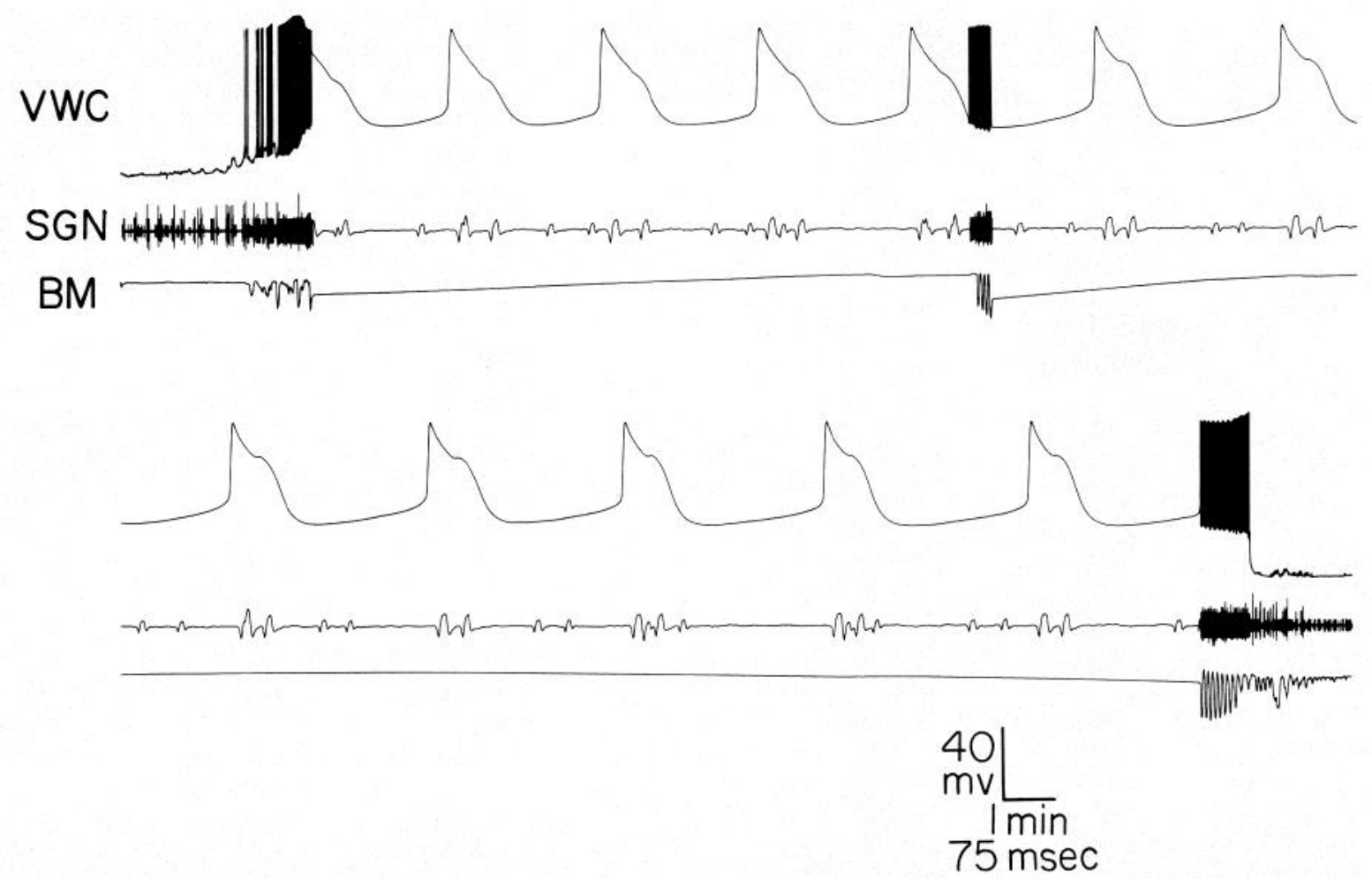

Figure 6. The broadened action potential coincides with behavioral effect and enhanced peripheral transmission during a prolonged burst. Continuous record of the VWC, SGN, and buccal mass was recorded as in Figure 4. At the time the buccal mass first exhibits extreme retraction (upward deflection of the force transducer) during stimulation by food, the VWC is at a point in its prolonged burst episode where the undershoot is most attenuated. The chart recorder speed was increased at this point to demonstrate the relationship between these buccal mass movements and the action potential waveform, which is now broadened with the characteristic hump on the repolarizing phase (Gillette et al, 1980) as compared with initial unbroadened spikes in these bursts. At this point in the burst the broadened somatic spike of the VWC is supporting two axonal spikes in the contralateral SGN.

agus is demonstrated in Figure 7. Direct stimulation of the VWC by intracellular microelectrode produces a graded contraction of the esophagus. The degree of contraction is directly related to the number and frequency of spikes in a train (Fig. $7 A$ ). As few as 5 spikes at $6 \mathrm{~Hz}$ will produce a measurable contraction. Direct stimulation at $5 \mathrm{~Hz}$ for $15 \mathrm{sec}$ produces extreme shortening (Fig. $7 A$ ), like that seen during VWC bursting stimulated by $\mathrm{SH}$ in the buccal cavity (Fig. $7 B$ ). When VWC bursting is activated by $\mathrm{SH}$ in the buccal cavity, the initiation of esophageal shortening takes longer than in the case of direct stimulation, possibly because the cell is firing at a lower frequency $(4 \mathrm{~Hz})$; but once contraction is initiated, it proceeds smoothly to extreme shortening (Fig. $7 B$ ). This shortened state is maintained at its extreme for only a few seconds, after which the esophagus begins slow relaxation, even though the cell continues to fire at a high frequency.

In the intact animal, the esophagus morphology is dramatically altered by ingestion of a large meal (Fig. 8). The esophagus in the unfed animal is a muscular, thickwalled tube (Fig. $8 A$ ), purple-brown in color, connecting the buccal mass with the stomach. Waves of peristalsis pass down the esophagus from its anterior origin at the dorsal side of the buccal mass to the gastroesophageal sphincter which separates the esophagus from the stomach. The lumen diameter of the esophagus varies, de- pending on the size of the animal, but is rarely greater than $0.5 \mathrm{~cm}$ prior to feeding.

In an animal fed to satiation on a SR, the SR is packed in coils filling the esophagus from the gastroesophageal sphincter right up to the buccal-esophageal sphincter, with no food in either the stomach or the buccal mass itself (Fig. $8 B$ ). This packing of the esophagus stretches the muscle wall to transparency; the coils of squid and their color may be clearly discerned. The esophagus is stretched to more than half the length of the animal and the maximum diameter of the esophagus in a 300 -gm animal may now be 5 to $6 \mathrm{~cm}$ across the ovoid distension. Within $3 \mathrm{hr}$ post-satiation the squid starts to move into the stomach. This process may take $24 \mathrm{hr}$ or more to complete. Initial stages of digestion take place here in the esophagus, for the ingested food mixes with secretions of both the salivary and acid gland (M. P. Morse, personal communication). Thus, during the consummatory phase of feeding, the esophagus may serve as a distensible storage organ, a pouch into which the food can be rapidly packed when it is available and stored in preparation for more complete digestion later.

\section{Buccal mass movements and VWC activity during chemosensory stimulation: Emetic responses}

The two classes of electrical responses to emetic stimuli observed in the VWC (Fig. 3) correlate with two 

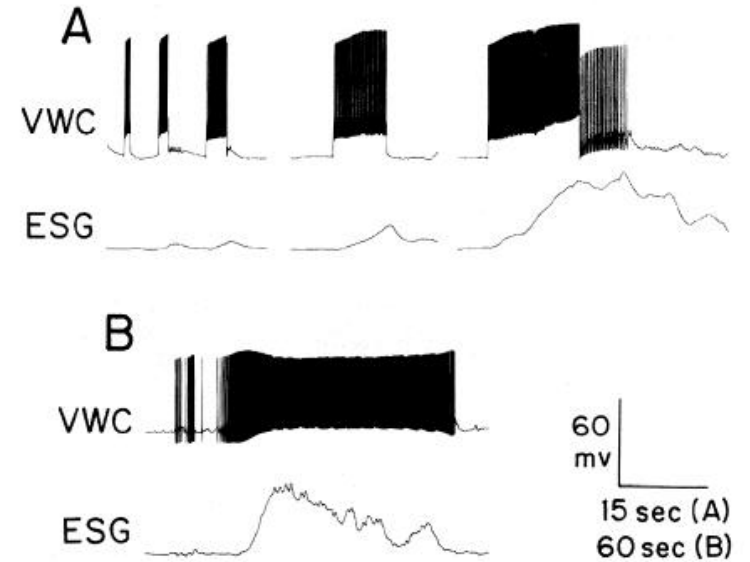

Figure 7. Response of the esophagus ( $E S G$ ) to VWC activity. $A$, Effect of direct intracellular stimulation of the VWC on the esophagus. In the first record groups of 5,10 , and 20 action potentials stimulated at $6 \mathrm{~Hz}$ in the VWC produce successively larger contractions of the esophagus, each with a slight delay. In the second record, a train of action potentials stimulated at $5 \mathrm{~Hz}$ for $9 \mathrm{sec}$ produces a larger esophageal contraction. There is a 2-sec delay from initiation of spiking to the onset of contraction. In the third record, high frequency stimulation produces extreme shortening of the esophagus. $B$, Effect on the esophagus of VWC activation by food. In a different experiment, prolonged endogenous bursting of the VWC was stimulated by $\mathrm{SH}$ introduced into the buccal cavity. Shortly after the onset of high frequency firing during the burst, during the initiation of vigorous ingestive buccal mass movements, the esophagus undergoes dramatic shortening. Maximal contraction is not maintained throughout the burst. In both $A$ and $B$ a force transducer was attached by a hook through the lateral edges of the cut distal end of the esophagus and adjusted to the length of the relaxed esophagus. The magnitude of contractions in $A$ and $B$ are not directly comparable due to differences in the size of the animals and the length of their esophagi.

distinct behavioral responses. One response was elicited by $\mathrm{EtOH}$ or Haemosol applied to the oral veil (2 of 6 animals) or buccal cavity (2 of 6 animals): the animal withdrew first its sensory papillae and then the oral veil, and contracted away from the stimulus; no buccal mass movements were observed. This behavioral response was accompanied by a slow, steady hyperpolarization of the membrane potential of the VWC which endured until stimulus washout (Fig. $3 A$ ).

In four of six animals, introduction of emetic substances into the buccal cavity produced vigorous movements of the buccal mass which we interpret as egestion (Fig. 9). The movements were distinct from those elicited by appetitive stimuli: the medial radular sac alternated between rest and protraction while the lateral lobes containing the projections of the jaws alternately moved medially, then laterally (i.e., sideways), rather than rolling under the radular sac as was the case in appetitive stimulation. Figure 9 shows that intense tonic activity in an otherwise unidentified unit in the contralateral SGN precedes and continues throughout the buccal mass movements. This unit is clearly distinct from the VWC which spikes infrequently, if at all, during emetic stimulation of the buccal cavity (Fig. 9). The functional relationship between tonic activity in this unit and eges- tion is unknown. During the egestive movements of the buccal mass, the membrane potential of the VWC begins to oscillate, in some cases in rhythm with the movements of the buccal mass (Fig. 9, line 2), but the oscillations subside before the brief period of VWC spiking which precedes hyperpolarization and silence in the VWC. Indeed, in the experiment shown in Figure 9, activity in the VWC and movements in the buccal mass ceased roughly 2 to $4 \mathrm{~min}$ after emetic stimulation. Both the cell and the buccal mass were inactive throughout stimulus washout. When animals were presented with food in the continued presence of emetic substances, the VWC was unresponsive and remained hyperpolarized. Thus, hyperpolarization of the VWC by emetic substances parallels a negative motivational state in the animal toward food.

\section{Activation of the VWCs by food parallels behavioral arousal}

Since the VWC became hyperpolarized and silent in response to emetic stimuli (after the egestion movements of the buccal mass ceased), we felt it was necessary to demonstrate reversal of the response to confirm that the preparation was still healthy. Every exposure to an emetic stimulus was reversible after careful washout and re-introduction of food, but each animal showed a higher threshold and a much greater latency from appetitive stimulus application to prolonged VWC bursting than it did at the time of initial food application prior to the aversive experience. The mean latency from the first application of SH after washout of emetic stimuli to a prolonged burst episode in the VWC was $15.45 \pm 6.53$ min (SD) in three different experiments. The period from the initial application of $\mathrm{SH}$ to prolonged bursting in these animals when they were naive and hungry at the beginning of the experiment averaged $3.32 \pm 1.02 \mathrm{~min}$ (SD). This difference is significant at the 0.02 level (twotailed $t$ test). Comparison of the interburst intervals for recurrent prolonged bursts before and after Haemosol inhibition of the VWC shows no significant difference; in fact, the intervals are overlapping in individual preparations. This demonstrates that the decline in activation rate of the VWC after Haemosol experience is not due to deterioration of the preparation. Thus the rate of activation of the VWC by appetitive stimuli varies with animals' behavioral histories, and the individual animal's chemosensory experience can clearly exert a much greater effect on its latency of response to food than phenotypic variations between hungry animals exert on their initial feeding responses. In each case, VWC activation parallels behavioral arousal by food, and arousal culminates in the vigorous feeding driven by prolonged burst episodes of this cell.

\section{Discussion}

Responses of the VWC to chemosensory stimuli. In undertaking the study of the pathways presynaptic to the VWC, we sought to establish the nature of chemosensory stimuli which are the extrinsic sensory activators of this neuron. Our results demonstrate a clear relationship between the chemosensory character of the stimulus and its effect on the VWC. In the unstimulated hemi- 
animal, the VWC is quiescent, exhibiting a relatively flat base line with little or no spiking (Fig. 2). Appetitive chemosensory stimuli, those that elicit ingestion of a neutral stimulus (string), when applied at normal chemosensory sites on the animal (oral veil or buccal cavity) cause gradual excitation of the VWC to prolonged burst episodes. This phenomenon we have termed activation. Activation parallels a general arousal of feeding behavior in the animal. Application of emetic stimuli-those that elicit egestion of the neural stimulus (string) when applied at normal chemosensory loci on the oral veil or buccal cavity - has the general effect of hyperpolarizing this cell, an effect which parallels a negative motivational state in the animal toward feeding. Thus appetitive stimuli, but not emetic stimuli, are the extrinsic activators of the VWC. Furthermore, activity in the VWC reflects a positive motivational state toward food. The details of these responses will be discussed in turn.

Appetitive stimuli consistently caused the VWC to shift (Fig. 2) from quiescence to synaptically driven activity which culminated in the endogenous prolonged burst episode. The prolonged burst episode has previously been demonstrated to be the endogenously driven command phase of this neuron's activity (Gillette et al., 1980). Application of food to the chemosensory oral veil or buccal cavity of the hemi-animal arouses spike activity in a quicscent VWC within $1 \mathrm{~min}$ (Fig. 2). We do not know whether the length of this period is increased over that in the intact animal by dissection or whether it reflects a real time necessary for arousal or disinhibition of the feeding circuits in an animal not primed to feed. ${ }^{3}$ However, once initiated, activation builds through excitatory synaptic activity: the VWC gradually depolarizes, the spike frequency increases, and the spike activity becomes patterned in short bursts. During this early phase of activation, the VWC is following cyclic activity of the network, co-active with retraction motor neurons (Gillette et al., 1980). Appetitive stimulation culminates in the prolonged burst episode, the fully activated state of this neuron. Once triggered, the prolonged burst episode is self-sustaining (Gillette et al., 1980). During the prolonged burst episode, the VWC drives the feeding network, increasing the cycling rate of the buccal mass and commanding intense output of motor neurons of the buccal retractor muscles (Fig. 4). These results corroborate our earlier work on the isolated ganglion (Gillette et al., 1980). An increase in the cycling rate of as much as 2.4-fold, extreme retraction of the radula, and a predominance of the duration of retraction phase so that the mean ratio of protraction to total cycle time is $0.41 \pm$ 0.04 per cycle characterize the changes in buccal mass activity during the driving period of the VWC burst.

Our present findings explicitly demonstrate that the behavior driven by the VWC is one of ingestion. We have demonstrated this in three different ways. First, prolonged burst episodes are stimulated in the VWC by appetitive stimuli but not emetic stimuli applied at nor-

\footnotetext{
${ }^{3}$ We have examined previous rccords for 54 animals that responded to $\mathrm{SH}$ with biting within $90 \mathrm{sec}$. Mean bite latency from onset of stimulation of the oral veil was $24.9 \mathrm{sec}(\mathrm{SD} \pm 22.9 \mathrm{sec}$ ); the range was 1.8 to $80.0 \mathrm{sec}$ (J. London and M. Gillette, unpublished observations).
}

mal chemosensory sites on the oral veil and buccal cavity. Second, the motor pattern stimulated by food and driven by VWC bursting is a distinctive one in which retraction predominates over the protraction phase of movement of the radula (Fig. 4). We have measured a mean ratio of protraction to total cycle time of $0.41 \pm 0.04$ (range 0.20 to 0.54 ) from force transducer records of complete feeding bursts compared with $0.40 \pm 0.08$ (range 0.30 to 0.50 ) reported by Croll and Davis (1981) from mid-feeding cinematographic records. This pattern has been shown to distinguish the feeding motor program from other rhythms of the buccal mass of Pleurobranchaea in both whole animal and reduced preparations (Croll and Davis, 1981, 1982). Finally, VWC bursting activated either by food in the mouth or imposed depolarization of the VWC actually causes movement of food from the buccal cavity into the esophagus during the feeding motor pattern (Fig. $5)$. Therefore, the command qualities of VWC bursting clearly pertain to feeding behavior.

Emetic stimuli applied to the oral veil or buccal cavity of the hemi-animal were unable to activate prolonged burst episodes (Table I). Such stimuli produced one of two responses: (1) slow, deep hyperpolarization of VWC membrane potential analogous to inhibition of long duration coincident with avoidance or withdrawal movements by the head region (Fig. 3) or (2) rhythmic oscillations of the membrane potential in phase with buccal mass movements which were distinct from feeding movements and are presumed to represent egestion (Fig. 9). These oscillations may arise from activity in some of the many neurons presynaptic to the VWC in the buccal ganglion, but they did not result in sustained action potential activity in the VWC and were never observed to stimulate prolonged burst episodes.

Indeed, emetic stimuli have a potent inhibitory effect on the long-term activity of the cell: they result in a deeply quiescent cell that is more resistant to activation by food stimuli. Without exception, emetics inhibit the cell by a slow hyperpolarization. We have observed VWCs in hemi-animals exposed to emetic stimuli for up to $1 \mathrm{hr}$; during this period and the subsequent wash they are profoundly quiet. Subsequently, it takes several applications of appetitive stimuli to elicit the first signs of VWC activation. The resulting activation is slower than that observed when a food-deprived animal is initially presented with food; it includes a long depolarization phase before spiking begins and the burst episode is longer than it is prior to emetic treatment $(n=3)$. Thus, the inhibition caused by emetic substances effectively parallels a behavioral state in which the animal is disinterested in or averse to food.

Relation to other studies. Our conclusions on the role of the VWC contradict interpretations of previous studies in which the VWC was thought to command egestion. McClellan (1980) attempted to distinguish between the neuronal bases of feeding and regurgitation in Pleurobranchaea. Initially, he identified the activity phases of selected muscles in the two motor programs in the behaving, semi-intact animal and concluded there were no differences in burst durations of protractors and retractors during ingestion and egestion. He then moved to more reduced preparations for the cellular analyses. 

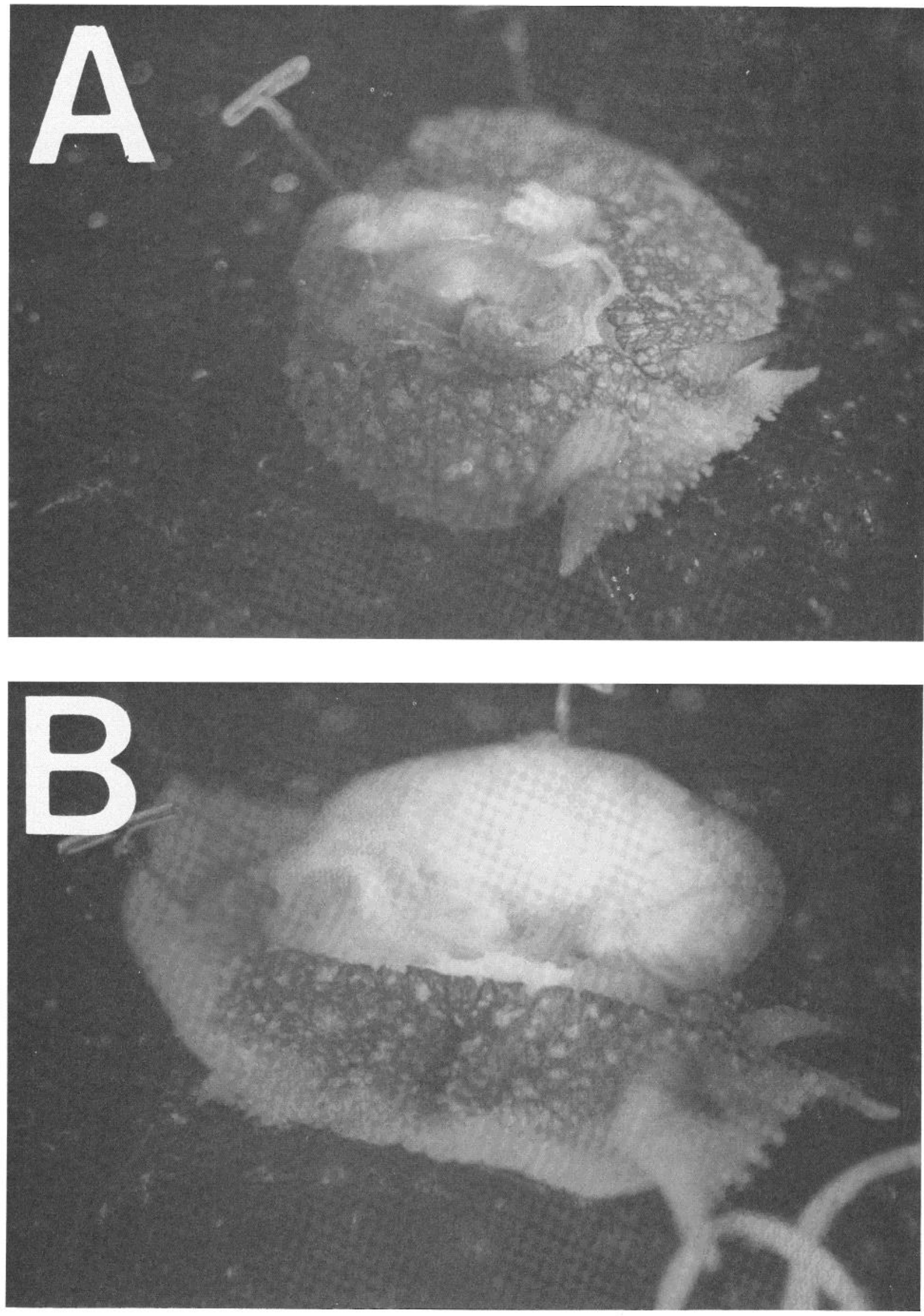

Figure 8. Response of the esophagus to the rapid ingestion of solid food. A, The esophagus in an unfed animal is visible through the mid-dorsal incision in the mantle as a cashew-shaped structure about $2 \mathrm{~cm}$ in length near the center of the animal 


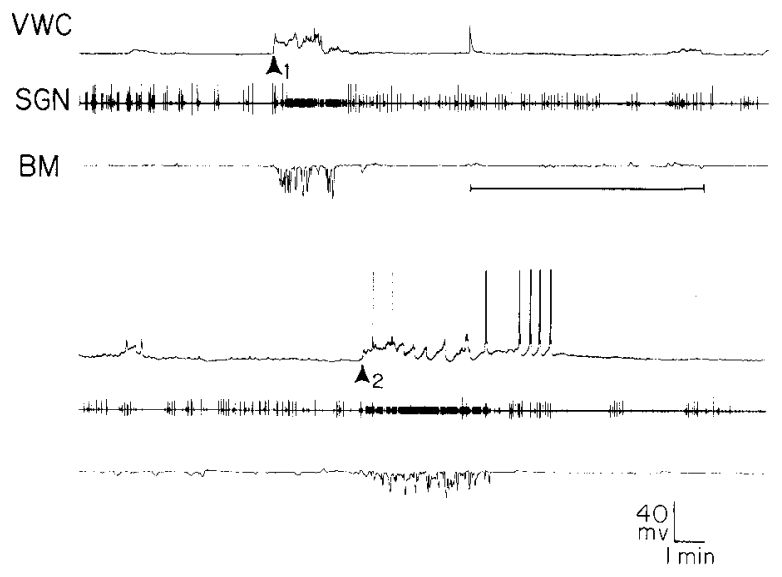

Figure 9. Buccal mass $(B M)$ movements and VWC activity: emetic responses. Records are from the same preparation as those in Figure 4. Emetic substances were introduced into the buccal cavity by cannula after the base line activity of the cell had been established. Buccal application of $10 \%$ EtOH (arrowhead 1) or 1\% Haemosol (pH 7.5; arrowhead 2) causes rhythmic oscillations in the membrane potential of the VWC, with occasional spikes in the case of Haemosol. During the period marked by the bar the cannula was extensively rinsed and fresh sea water was exchanged for one-sixth of the fluid in the preparation dish. The radular sac of the BM alternates between rest and protraction (downward deflection of the force transducer) during the first minutes after emetic stimulus application. Axonal VWC activity is monitored in the contralateral SGN. Continuous record.

However, McClellan's reduced preparations would not ingest food; thus the ingestive part of the motor pattern was not examined at the level of patterned activity in the nerve roots. What he did study was peristaltic behavior of the esophagus, which he termed "swallowing," observing the peristaltic movement of pieces of squid introduced into the esophagus from the cut distal end. Thus, his force transducer records show little or no radular movement, nor did he find that food introduced to the esophagus stimulated the VWC. We have found that the completely denervated esophagus will exhibit peristalsis when SH is placed inside; therefore, this swallowing of small amounts of food is at least in part peripherally mediated (unpublished observations). Therefore, McClellan's study of feeding behavior differs from ours in that McClellan studied a swallowing phase and we have studied the ingestive phase.

McClellan's study of egestion differs from ours for similar reasons. He did not look at the effect of emetics introduced to the oral veil or buccal cavity. We have addressed this question and found that the VWC is not activated to burst by noxious substances on the oral veil or during their expulsion from the buccal cavity. McClellan looked only at the behavioral response to soap
(Ivory) placed in the esophagus from the cut distal end: this caused intense cycling of the radula and the buccal motor roots during egestion of soap from the esophagus (regurgitation). He did not record from the VWC during this behavior but moved to the isolated nervous system for his neuronal studies. As the emetic stimuli introduced via the mouth in our experiments caused immediate egestion of the substance, we suspect that it did not reach the esophagus, nor would it in the feeding whole animal. Therefore, our study did not overlap with this aspect of McClellan's work either.

McClellan looked at the cycling rate of buccal roots 1 and 3 to both SGN stimulation (McClellan, 1980, 1982b) and VWC stimulation (McClellan, 1980) in the isolated nervous system. He found that in both cases he elicited intense alternating bursts in the roots. He concluded that this represented regurgitation because the only time he saw intense cycling in his more intact behaving preparations was during egestion. Our results agree with those of Croll and Davis (1981): that intense cycling is also characteristic of the ingestive phase of feeding.

McClellan's analysis, revealed in summary diagrams, determined no difference in the duration of muscle bursts between retractors and protractors of the semi-intact preparation (McClellan, 1980; see Fig. 8 in McClellan, 1982a) or in cut motor nerve roots of the reduced preparation (McClellan, 1980; see Fig. 6 in McClellan, 1982b); thus, he did not use the patterned muscle or nerve root activity to distinguish ingestion and egestion. However, inspection of his records clearly shows that during egestion, protraction bursts dominate (McClellan, 1980; see Figs. 2 to 4, McClellan, 1982a), as Croll and Davis (1981) found. The one figure of consummatory feeding shown provides too little data to assess the predominance of retraction during ingestion (McClellan, 1980; Fig. 6 in McClellan, 1982a). Instead of the motor program pattern, he used electrical inactivity in the salivary duct as a criterion to distinguish regurgitation. However, he observed that the salivary duct can be inactive during swallowing (McClellan, 1980, 1982a) and also showed that repetitive VWC activity and salivary duct activity can occur together (Figs. 5-11 and 5-15 in McClellan, 1980).

Croll and Davis (1981) convincingly showed that the vigorous cycles of feeding and regurgitation differ in the relative durations of root 3 bursts (retraction) to root 1 bursts (protraction): root 3 bursts predominate during ingestion cycles and root 1 bursts predominate during egestion cycles. Our observations confirm their data. In those records of McClellan's where cyclic bursts were stimulated in the isolated nervous system by SGN or VWC stimulation, the duration of root 3 exceeds root 1 in every case (Figs. 4-8, 4-9, 4-10, 5-8, 5-10,5-12,5-13, McClellan, 1980; Fig. 8-10, McClellan, 1982b). This suggests that these motor patterns actually represent inges-

in this picture. The constriction at the posterior end of the esophagus is the gastroesophageal sphincter. $B$, In an animal fed to satiation on SR, the esophagus is the large ovoid structure protruding through the mid-dorsal incision. The esophagus is packed with ingested SR from the buccal-esophageal sphincter to the gastroesophageal sphincter and is stretched to about $6 \mathrm{~cm}$. The whitish coils of packed SR can be clearly seen through the now transparent esophageal wall. An uningested length of SR lies in the lower right of the picture. This 200 -gm animal consumed $13.06 \mathrm{gm}$ of squid, a $127-\mathrm{cm}$ length of SR, in $4 \mathrm{~min}$ shortly before this picture was taken. After photographing, the animal was then returned to our sea water system and observed for 24 hr during which time the SR began to move into the stomach; none of this SR was egested. The cross-bar on the T-pins is $1.5 \mathrm{~cm}$ in length. 
tion, rather than egestion, and are consistent with our interpretation of the role of the VWC.

McClellan raises the idea that the VWC may be activated at the level of the esophagus to initiate regurgitation, but he did not record from the VWC in other than the isolated nervous system. We have not addressed this subject in the present study. What we have shown is that the VWC does drive ingestion but does not drive egestion of noxious substances from the buccal cavity of the animal. The notion that the VWC plays a command role in more than one behavior is provocative and deserves further examination.

Recently, Croll and Davis (1982) also suggested that the VWC is a central switch or trigger for egestion. This conclusion was based on a study of ingestion and egestion in a deafferented reduced preparation that did not respond behaviorally to chemosensory stimuli. They looked at movement of a neutral object caused by repetitive stimulation of the SGN; such stimulation drives cyclic motor output in the buccal ganglion. Their records show intense activity in some SGN unit(s) in the SGN contralateral to the stimulated nerve during egestion. The authors inferred that that tonic unit was the VWC because the VWC has an axon in that nerve and is known to fire in a similar fashion. However, they did not identify the VWC by intracellular recording. The behavior studied in our experiments was elicited by chemosensory stimuli applied at normal chemosensory sites while recording directly from the neuron in question. Our data indicate that, although a unit(s) in the SGN is tonically active during egestion, it is not the VWC (Figs. 2 and 9).

Croll and Davis (1982) suggested that Figure 3 by Gillette et al. (1980) indicates that the VWC drives egestion on the basis that burst durations in root 3 (retraction motor neurons) driven by the VWC were shorter than bursts in root 1 (protraction). However, we believe that in that figure the record is too slow to easily discern root 3 burst durations during the intense activity driven by the VWC. The only aspect of those root 3 bursts that stands out is intense firing in one or more of the largest units; the duration of that particular activity in the retraction burst is fractional and would cause underestimation of the total root 3 retraction burst duration. This large unit(s) appears to be present in the root 3 bursts shown in the Figure 5 of Croll and Davis (1982); the unit(s) drop out of the root 3 bursts recorded during an egestion episode. Furthermore, in Figure 2 of Gillette et al. (1980) a more easily measurable record of activity driven by the VWC is shown. Here, the averaged duration of the bursts recorded intracellularly in a root 3 retraction motor neuron is about $55 \%$ of the cycle period; protraction activity observed in the upper trace of that record is much less. Other of our unpublished records are consistent. The activity driven by the VWC satisfies criteria used by Croll and Davis (1981) to identify the motor rhythm of feeding behavior.

Mechanism of VWC activation. Appetitive stimuli at both the oral veil and buccal cavity can activate the VWC; however, food in the buccal cavity is much more effective than food on the oral veil in stimulating prolonged burst episodes (Table II). This would suggest that the major role of the VWC is in the late consummatory phase of feeding, rather than in the appetitive phase. This may leave initiation of feeding in Pleurobranchaea to neurons in the cerebral ganglion (Gillette et al., 1982c), while vigorous consummatory command resides in the buccal ganglion, a functional separation like that demonstrated for Aplysia by lesion studies (Kupfermann, 1974b). Buccal excitation culminates in prolonged burst episodes in the VWC, which drive vigorous, extreme oscillations of the buccal mass. During the prolonged burst episode the VWC commands the final phase of consummatory behavior, the rapid ingestion of food. A single such bout of ravenous bolting would be sufficient for the animal to ingest moderate-sized prey as we have observed the animal to consume, including ectoprocts and nudibranchs, as well as a considerable amount of squid flesh (Davis et al., 1977). The continuous presence of food in the buccal cavity elicits recurrent bouts of prolonged burst episodes in the VWC and attendant ingestive behavior (Table II, Fig. 4). The presence of chemoreceptors in the mouth suggests that the palatability of the substance is reassessed before vigorous ingestion occurs, as is the case in Limax (Gelperin et al., 1978). Thus, Pleurobranchaea is aroused to more vigorous ingestive feeding behavior by the arrival of food in the mouth, a conclusion which resembles that made by Kupfermann (1974a) for Aplysia.

Our earlier work on the isolated buccal ganglion demonstrated that treatments which elevate intracellular cAMP rapidly activate the VWC (Gillette, M. U., et al., 1978; Gillette, R., et al., 1982b). Appetitive stimulation at the buccal cavity or oral veil of the hemi-animal produces the same activation of prolonged burst episodes as the variety of cAMP treatments (Gillette, M. U., 1981). Furthermore, burst episodes stimulated by food at chemosensory sites resemble in detail the electrical changes induced by elevated intracellular cAMP within the VWC (M. Gillette, submitted for publication). These findings suggest that food stimulation of the buccal cavity activates a neuromodulatory pathway which stimulates cAMP accumulation within the VWC. Accumulation of cAMP above a certain threshold level would induce prolonged bursting through potentiation of slow inward membrane currents (Gillette, R., 1981, submitted for publication). These possibilities are currently under investigation.

Mechanism by which the VWC commands feeding motor output. The evidence that the VWCs actually serve a command function in feeding behavior may be summarized as follows. First, the neurons drive ingestive motor activity during trains of broadened action potentials; such trains occur during the prolonged and sustained burst episodes. Second, the capacity to support such burst episodes, accompanied by ingestive behavior, is induced by chemosensory stimulation of the animal with normally appetitive stimuli. One final piece of documentation which has not yet been obtained from the hemianimal preparations we used is to hyperpolarize the neurons during the food-stimulated burst episode order to observe the expected slowing of feeding activity. While this measurement is desirable (cf. Kupfermann and Weiss, 1978), technical constraints have so far prevented its performance; for instance, the necessity for paired 
electrode impalements of the VWCs in a ganglion; the probability that both VWCs will be located on the ganglion surface in any particular preparation is only about $25 \%$. Such experiments have been performed in the isolated ganglion with the result that imposed termination of spontaneous burst episodes is followed by reduction or cessation of motor output (unpublished data), and there seems little reason to suppose that the eventual successful performance in the hemi-animal will have a contradictory outcome.

Experiments in which the soma was hyperpolarized while driving the cell antidromically via its axon also suppressed motor output (Gillette et al., 1980), indicating that spike frequency alone does not drive the command functions of the VWC. In the present studies the effect of the VWC on feeding cycle frequency and intensity becomes apparent when the waveform of the spike has significantly broadened (Fig. 6). This agrees with our earlier finding that it is not so much through spike frequency but rather through spike broadening that the VWC commands the increased motor output of the isolated buccal ganglion (Gillette et al., 1980). In the soma, and presumably in the dendrites, the prolonged burst and spike broadening are in part caused by enhanced inward $\mathrm{Ca}^{2+}$ currents which may drive these central effects of the VWC on the network by augmenting transmitter release (Gillette et al., 1982a; R. Gillette, submitted for publication).

The heightened effects exerted on the esophagus during intense bouts of feeding are mediated by a basically different mechanism. Unlike the soma spike, the VWC axon spike does not broaden (Gillette et al., 1980), presumably because, as in other molluscan axons, the $\mathrm{Ca}^{2+}$ current is not appreciable (Junge and Miller, 1974). However, the broadening of the soma spike may be encoded in the axon spike frequency as is shown in Figure 6 . This is presumably because the increase in duration of the broadened somatic spike outlasts the refractory period of the first axonal spike and a second spike is initiated in the axon. Thus the broadened somatic spike acts to increase the cell's frequency output to the esophagus at the time it enhances cyclic motor activity via broadened spikes at dendritic sites in the buccal ganglion.

Behavioral significance of the VWC. The arrival of food at the oral veil or buccal cavity stimulates the feeding network, which the VWC initially follows, firing cyclically (Fig. 2). During this time, the radular sac of the buccal mass moves from rest to protraction and back to rest (Fig. 4), an action which pulls food into the mouth. With the induction of the prolonged burst episode, the VWC drives intense, cyclic movements of the radular sac, which now moves from protraction, where the radula engages the food, to extreme retraction, where it pauses before swinging forward again. The pattern of movement of the SR during VWC-driven buccal mass movements (Fig. 5) indicates that this pause represents a period where the food is disengaged from the hooked denticles of the radula in preparation for the return movement. The slight movement of the SR back toward the buccal mass from the esophagus while the radula is retracted (Fig. $5 B$ ) means that the SR is still attached to the denticles. Denticle disengagement must take place at the pause, for the ingested length is pushed into the esophagus as the radula is protracted to engage a new length. During these extreme movements, the cycling frequency of the buccal mass is nearly $2^{1 / 2}$ times that observed prior to the induction of prolonged VWC bursting. Thus with the increased radular excursion coupled to the increased cycling frequency, food is very rapidly ingested during the VWC burst period.

In unpublished observations on the whole animal (M. Gillette), ingestion of long ribbon cut from squid mantle ( 2 to $3 \mathrm{~m}$ ) was found to involve uptake of a regular, discrete length of material with each cycle of the radula. In a 300-gm Pleurobranchaea, this could involve ingestion of $1.5 \mathrm{~cm}$ of the SR/cycle, a length approximately equal to the total excursion of the radula. During ingestion of solid food like the SR, the buccal mass cycles at a frequency overlapping the range observed for ingestion of liquid food in these studies ( 18 to $28 \mathrm{cpm} /$ first minute in four animals). This means that a large bulk of solid food accumulates quickly during rapid ingestion. Upon dissecting animals immediately after feeding to satiation on SR, all of the squid is found packed in the very swollen esophagus, looped in great coils, and has not yet entered the stomach (as in Fig. 8). Distention of the anterior gut has been shown to mediate satiation in Aplysia (Susswein and Kupfermann, 1975). Our results, as well as those of Davis et al. (1977), are consistent with such a mechanism operating in Pleurobranchaea.

These observations provide a basis for a functional explanation for the output of the VWC to the esophagus during its activation by food (Fig. 6). The vigorous longitudinal contracture (Fig. 7) and circular dilation of the esophagus, coupled with tonic opening of the buccalesophageal sphincter during the VWC burst, ensure that food is efficiently packed into this storage organ from distal end first. If such contracture did not occur, a load would be created by food accumulating in the proximal end that must be pushed distally by incoming food. This burden would tend to impair the swallowing function of the buccal mass. The gradual relaxation of the esophagus during the burst (Fig. $7 B$ ) is consistent with accommodation of incoming food by the gradual distension of the esophagus. By this interpretation, the command role of the VWCs is thus a distinctive one which drives vigorous ingestion coordinated with visceral receptivity for a large meal. The command functions of the VWCs therefore may confer in a large part the behavioral qualities of opportunistic gluttony typical of Pleurobranchaea and many other predatory carnivores.

\section{References}

Croll, R. P., and W. J. Davis (1981) Motor program switching in Pleurobranchaea. I. Behavioral and electromyographic study of ingestion and egestion in intact specimens. J. Comp. Physiol. 145: 277-287.

Croll, R. P., and W. J. Davis (1982) Motor program switching in Pleurobranchaea. II. Ingestion and egestion in the reduced preparation. J. Comp. Physiol. 147: 143-153.

Davis, W. J., and G. J. Mpitsos (1971) Behavioral choice and habituation in the marine mollusk Pleurobranchaea californica MacFarland (gastropoda, opisthobranchia). Z. Vgl. Physiol. 75: 207-232.

Davis, W. J., M. V. S. Siegler, and G. J. Mpitsos (1973) 
Distributed neuronal oscillators and efference copy in the feeding system of Pleurobranchaea. J. Neurophysiol. 36: 258274.

Davis, W. J., G. J. Mpitsos, J. M. Pinneo, and J. L. Ram (1977) Modification of the behavioral heirarchy of Pleurobranchaea. I. Satiation and feeding motivation. J. Comp. Physiol. 117: 99-125.

Fretter, V., and A. Graham (1962) British Prosobranch Mollusks; Their Functional Anatomy and Ecology, Vol. 144, Ray Society, London.

Gelperin, A., J. J. Chang, and S. C. Reingold (1978) Feeding motor program in Limax. I. Neuromuscular correlates and control by chemosensory input. J. Neurobiol. 9: 285-300.

Gillette, M. U. (1981) Appetitive stimuli arouse the ventral white cell to the prolonged bursting and spike broadening that drive cyclic buccal mass movements in Pleurobranchaea. Soc. Neurosci. Abstr. 7: 642.

Gillette, M. U., R. Gillette, and W. J. Davis (1978) Cyclic AMP may modulate prolonged endogenous bursting and spike broadening in the ventral white cell of Pleurobranchaea. Soc. Neurosci. Abstr. 4: 1209.

Gillette, R. (1981) Comparison of the effects of cyclic AMP and $\mathrm{NH}_{4}{ }^{+}$on the electrophysiology of the ventral white cell of Pleurobranchaea. Soc. Neurosci. Abstr. 7: 113.

Gillette, R., and W. J. Davis (1977) The role of the metacerebral giant neuron in the feeding behavior of Pleurobranchaea. J. Comp. Physiol. 116: 129-159.

Gillette, R., M. U. Gillette, and W. J. Davis (1978a) Prolonged endogenous bursting and spike broadening are substrates of command function in the feeding network of Pleurobranchaea californica. Soc. Neurosci. Abstr. 4: 1210.

Gillette, R., M. P. Kovac, and W. J. Davis (1978b) Command neurons in Pleurobranchaea receive synaptic feedback from the motor network they excite. Science 199: 798-801.

Gillette, R., M. U. Gillette, and W. J. Davis (1980) Action potential broadening and endogenously sustained bursting are substrates of command ability in a feeding neuron of Pleurobranchaea. J. Neurophysiol. 43: 669-685.

Gillette, R., M. U. Gillette, and W. J. Davis (1982a) Substrates of command ability in a buccal neuron of Pleurobranchaea. I. Mechanisms of action potential broadening. J. Comp. Physiol. 146: 449-459.

Gillette, R., M. U. Gillette, and W. J. Davis (1982b) Substrates of command ability in a buccal neuron of Pleurobranchaea. II. Potential role of cyclic AMP. J. Comp. Physiol, 146: 461470.
Gillette, R., M. P. Kovac, and W. J. Davis (1982c) Control of feeding motor output by paracerebral neurons in the brain of Pleurobranchaea californica. J. Neurophysiol. 47: 885-908.

Hirsch, G. C. (1915) Die Ernahrungsbiologie Fleischfressender Gastropoden (Murex, Natica, Pterotrachaea, Pleurobranchaea, Tritonium). I. Teil. Macroskopischer Bau, Nahrungsaufnahme, Verdauung, Sekretion. Zool. Jahrb. Abt. Allg. Zool. Physiol. Tiere 35: 357-504.

Junge, D., and J. Miller (1974) Different spike mechanisms in axon and soma of molluscan neurons. Nature 252: 155-156.

Kupfermann, I. (1974a) Feeding behavior in Aplysia: A simple system for the study of motivation. Behav. Biol. 10: 1-26.

Kupfermann, I. (1974b) Dissociation of the appetitive and consummatory phases of feeding behavior in Aplysia: A lesion study. Behav. Biol. 10: 89-97.

Kupfermann, I., and K. R. Weiss (1978) The command neuron concept. Brain Behav. Sci. 1: 3-39.

Lee, R. M., and R. J. Liegeois (1974) Motor and sensory mechanisms of feeding in Pleurobranchaea. J. Neurobiol. 5: $545-564$

Lee, R. M., M. R. Robbins, and R. Palovcik (1974) Pleurobranchaea behavior: Food finding and other aspects of feeding. Behav. Biol. 12: 297-315.

McClellan, A. D. (1979) Swallowing and regurgitation in the isolated nervous system of Pleurobrachaea: Distinguishing features and higher order control. Soc. Neurosci. Abstr. 5: 830.

McClellan, A. D. (1980) Feeding and regurgitation in Pleurobranchaea californica: Multibehavioral organization of pattern generation and higher order control. Ph.D. Thesis, Case Western Reserve University.

McClellan, A. D. (1982a) Movements and motor patterns of the buccal mass of Pleurobranchaea during feeding, regurgitation and rejection. J. Exp. Biol. 98: 195-211.

McClellan, A. D. (1982b) Re-examination of presumed feeding motor activity in the isolated nervous system of Pleurobranchaea. J. Fxp. Biol. 98: 213-228.

Reingold, S. C., and A. Gelperin (1980) Feeding motor program in Limax. II. Modulation by sensory inputs in intact animals isolated central nervous systems. J. Exp. Biol. 85: 1-19.

Susswein, A. J., and I. Kupfermann (1975) Localization of bulk stimuli underlying satiation in Aplysia. J. Comp. Physiol. 101: 309-328.

Willows, A. O. D. (1978) Physiology of feeding in Tritonia. I. Behavior and mechanics. Mar. Behav. Physiol. 5: 115-135. 